\title{
Prevalence of the CHEK2 R95* germline mutation
}

\author{
Stian Knappskog ${ }^{1,2^{*}}$, Beryl Leirvaag ${ }^{1,2}$, Liv B. Gansmo ${ }^{1,2}$, Pål Romundstad ${ }^{3}$, Kristian Hveem³ ${ }^{3}$ Lars Vatten ${ }^{3}$ \\ and Per E. Lønning ${ }^{1,2}$
}

\begin{abstract}
Background: While germline CHEK2 mutations have been linked to a moderately elevated cancer risk, to date, a limited number of such mutations have been identified. Recently, we reported a germline nonsense mutation (C283T; R95*), introducing an early stop-codon, in two Norwegian patients diagnosed with locally advanced breast cancer. Both patients were resistant to anthracycline therapy, resembling what has been observed for TP53 mutations.
\end{abstract}

Methods: In the present study, we screened a large population based sample, including 3748 non-cancer individuals and 7081 incident cancer cases (breast cancer, $n=1717$; prostate cancer $n=2501$, lung cancer $n=1331$ and colorectal cancer $n=1532)$, for the distribution of CHEK2 R95*.

Results: We found that 12 individuals $(0.11 \%)$ carried the R95* variant: 4 non-cancer individuals $(0.11 \%), 4$ breast cancer cases $(0.23 \%)$, and 4 prostate cancer cases $(0.16 \%)$. Although the low number of observations precluded formal statistical assessment, our data may indicate an elevated risk for breast (OR: 2.19, 95 \% Cl: 0.55-8.75) and prostate cancer (OR: 1.5, $95 \%$ Cl: 0.36-6.00) associated with CHEK2 R95*. By mining international databanks, we found no individuals carrying the R95* mutation, indicating it to be restricted to the Norwegian population.

Conclusion: We provide proof-of-concept that previously unknown CHEK2 germline mutations may be present in certain populations. Notably, germline mutations in tumours are in general missed by contemporary massive parallel sequencing strategies, since tumour mutations are usually filtered against the germline. The fact that the CHEK2 R95* mutation may be associated with resistance to anthracyclines in cancer patients emphasizes its possible clinical importance.

\section{Background}

Checkpoint kinase 2 (CHEK2) has a critical role as a tumour suppressor, activating p53 in response to genotoxic stress. A limited number of germline point mutations in CHEK2 have been identified. In general, these are present at low frequencies and have moderate impact on cancer risk: the most frequent and most studied variant, the 1100delC truncation, occurs in approximately $1 \%$ of Northern Europeans [1-3]. Unlike single amino acid substitutions, the $1100 \mathrm{delC}$ truncation variant has been associated with an increased risk for cancer of the breast and prostate [1, 3]. Also, a larger $5395 \mathrm{bp}$

\footnotetext{
* Correspondence: stian.knappskog@uib.no

${ }^{1}$ Section of Oncology, Department of Clinical Science, University of Bergen, 5020 Bergen, Norway

2Department of Oncology, Haukeland University Hospital, Bergen, Norway Full list of author information is available at the end of the article
}

germline deletion observed in individuals of Eastern European descent, has been linked to increased risk of breast cancer [1, 4, 5], and recently several germline truncating CHEK2 mutations were linked to risk of Non-Hodgkin Lymphoma [6]. Taken together, these, and other data clearly indicates CHEK2 to be a multi-cancer susceptibility gene, with truncating mutation being of particular importance for some cancer types [7].

In a previous study, we found two unrelated patients to harbour a germline $\mathrm{C}-\mathrm{T}$ transition in position 283 of the coding region of CHEK2 (rs587781269; Fig. 1). This transition introduced a novel translation stop at codon 95 (CGA $\rightarrow$ TGA; R95*) causing a severely truncated protein [8]. The mutant protein proved to be non-functional both in terms of kinase activity and dimerization in in vitro assays [8]. Importantly, both patients carrying the $\mathrm{R}^{\circ}$ * variant revealed primary 


\section{CHEK2}

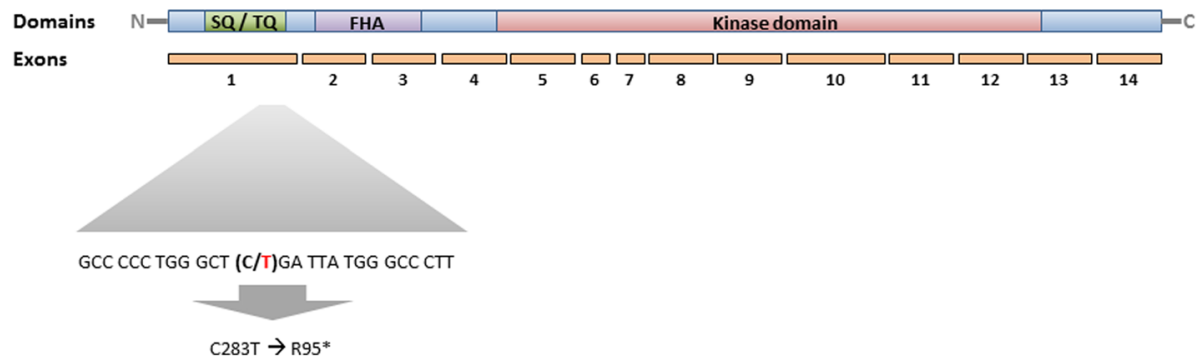

Fig. 1 Schematic representation of the CHEK2 gene with details on the flanking region of the R95* mutation (in bold; C= reference allele, $\mathrm{T}=$ alternative allele)

resistance to anthracycline therapy [8], resembling what we observed for patients with tumours harbouring somatic TP53 mutations in the same study [8-10].

The aim of the present study was to determine the CHEK2 R95* mutation incidence and potential influence on cancer risk across the general population.

\section{Methods}

\section{Study population}

All samples (DNA from blood) analyzed $(n=10,830)$ were obtained from the population-based Cohort of Norway (CONOR) study [11]. Participants were from all regions of Norway, but the majority $(n=4131)$ were residents of Nord-Trøndelag County in Mid-Norway. The mean age at sampling was 61.2 years (range 20.093.6 years). Incident cancers were identified by linking the identity of individuals to the Norwegian Cancer Registry. We analyzed 1717 incident cases of breast cancer, 1532 colon cancers, 1331 lung cancers and 2501 prostate cancers, in addition to 3749 control individuals without cancer. The 10,830 individuals are identical to the sample set previously described [12]. One sample failed analysis, thus, samples from 10,829 individuals were used for data assessments. All sample donors provided written informed consent to anonymous genetic testing for scientific purposes, and the study was approved by the Regional Committee for Ethics in Medical Research (REK Midt-Norge).

\section{CHEK2 R95* genotyping}

All samples were genotyped for the CHEK2 R95* mutation using a custom LightSNiP assay (TIB MOLBIOL Syntheselabor GmbH, Berlin, Germany) on a LightCycler 480 II instrument (Roche, Basel, Switzerland). Reaction mixes and thermocycling conditions were set according to the manufacturer's instructions

\section{Results}

In order to assess the frequency of the CHEK2 R95* mutation in the general Norwegian population, we genotyped peripheral blood DNA from a previously described [12] sample set $(n=10,829)$ extracted from the population based CONOR biobank. The CHEK2 R95* mutation was observed in four out of 3748 individuals without any cancer diagnosis $(0.11 \%$; Minor allele frequency [MAF] $5.3 \times 10^{-4}$; Table 1 ), in four out of 1717 breast cancer patients $(0.23 \%)$, and in four out of 2501 prostate cancer patients $(0.16 \%)$. In contrast, none out of 1331 lung cancer and none among 1532 colorectal cancer patients harboured the mutation (Table 1). While the low number of observations precluded any formal statistical assessment of the role of $\mathrm{R}^{\circ} 5^{*}$ as a potential cancer risk factor, our data may indicate an elevated risk for breast (OR: 2.19, 95 \% CI: $0.55-8.75$ ) and prostate cancer (OR: 1.5, 95 \% CI: 0.36-6.00) among mutation carriers. Supporting this, we found the age at diagnosis among the breast and prostate cancer patients to be

Table 1 Frequency of the CHEK2 R95* germline mutation in the Norwegian population

\begin{tabular}{|c|c|c|c|c|}
\hline Individuals/patients & R95*-carriers $^{\mathrm{a}} n$ & Wild-type $n$ & Total & MAF \\
\hline Non-cancer individuals & 4 & 3744 & 3748 & $5.3 \times 10^{-4}$ \\
\hline Cancer patients & 8 & 7073 & 7081 & $5.6 \times 10^{-4}$ \\
\hline Breast cancer & 4 & 1713 & 1717 & $1.2 \times 10^{-3}$ \\
\hline Prostate cancer & 4 & 2497 & 2501 & $8.0 \times 10^{-4}$ \\
\hline Lung cancer & 0 & 1331 & 1331 & 0 \\
\hline Colorectal cancer & 0 & 1532 & 1532 & 0 \\
\hline Total & 12 & 10,817 & 10,829 & $5.5 \times 10^{-4}$ \\
\hline
\end{tabular}

${ }^{\mathrm{a}}$ All R95* carriers were heterozygous 
slightly lower among the R95* carriers than among the CHEK2 wild-type patients (Table 2).

We performed data mining in order to assess whether the R95* mutation was restricted to certain geographical areas: We searched the 1000 genomes project phase 3, providing genomic data for 2439 individuals of different ethnic origin including several Caucasian sub-populations [13]: none of them harboured the R95* mutation, indicating that the variant may be restricted to the Norwegian population. The CONOR biobank contains samples collected from individuals living in distinct parts of Norway; thus, some regions are represented in particular. Accessing information about the county of residence for each individual donor, we found 11 out of 12 individuals harbouring the R95* mutation to be residents of the Nord-Trøndelag County in Mid-Norway (consisting of about $2.6 \%$ of the Norwegian population and contributing 4131 individuals to our cohort), contrasting one R95* mutation carrier out of 6698 participants living in other parts of Norway, (difference in incidence: $p=1.9 \times 10^{-4}$ ). Restricting the association estimates to residents of NordTrøndelag did not have any major impact on the results (OR: 1.49, 95 \% CI: 0.33-6.66 for breast cancer and OR: 1.32, 95 \% CI: $0.33-5.30$ for prostate cancer).

\section{Discussion}

In the present study, we screened a large population based cohort, and found the prevalence of the CHEK2 R95* mutation to be low and restricted to Norway.

Despite the low frequencies of the individual mutations, precluding proper statistical assessment of the odd ratios, germline CHEK2 mutations seem to be the underlying cause of hereditable cancer syndromes in some families $[1,2,14]$. To date, the most studied CHEK2 germline mutation is the $1100 \mathrm{delC}$ variant, which has a relatively high frequency in the Caucasian population: approximately $1 \%$ of healthy individuals are estimated to carry the 1100delC-variant [1-3]. Contrasting the risk assessments of single amino acid substitutions of the CHEK2, it has been estimated that the 1100delC-variant confers a two-fold elevated risk of both breast cancer [3] and prostate cancer [1]. Further, another deletion variant in Eastern Europe (5395 bp deletion) has also been linked to increased breast cancer risk $[1,4,5]$ and several germline truncating variants have been linked to Non-Hodgkin lymphoma [6].

Recently, another novel germline CHEK2 mutation, Y390C, was reported [15]. Interestingly, this mutation

Table 2 Age at cancer diagnosis

\begin{tabular}{llll}
\hline \multirow{2}{*}{ Diagnosis } & \multicolumn{3}{l}{ Average age at cancer diagnosis } \\
\cline { 2 - 4 } & R95*-carriers & Wild-type & Total \\
\hline Breast cancer & 55.75 & 60.36 & 60.35 \\
Prostate cancer & 69.75 & 71.72 & 71.72 \\
\hline
\end{tabular}

was associated with elevated risk for breast cancer but also resistance towards anthracyclines in vitro. In an earlier study, we also reported another truncating CHEK2 mutation, (ins1368A), to be associated with resistance to anthracycline therapy in a breast cancer patient [16]; thus, all these three mutations (R95*, Y390C and ins1368A) seem associated with defect DNA damage signaling leading to drug resistance. Notably, this contrasts some of the previously reported missense mutations (e.g. I157T and I364T) that seem to retain a wildtype kinase activity [8]. While we may not draw any final conclusion due to a limited number of observations, our data are consistent with the hypothesis [1-5] that certain CHEK2 mutations (as opposed to most missense mutations) may be associated with elevated cancer risk and, more importantly, resistance towards DNA damaging agents used in cancer therapy [9].

Our findings underline some issues that need to be taken into consideration. Firstly, most likely, there are additional germline pathogenic CHEK2 mutations, not yet discovered. These mutations are likely to be rare and may be distributed across restricted geographical areas. Secondly, such mutations may be of severe clinical importance, e.g. have an impact on cancer risk and sensitivity to chemotherapy $[8-10,16]$. Thirdly, as massive parallel sequencing strategies are increasingly being used, not only for research purposes, but also to guide treatment decisions, it is important to note that such germline mutations are likely to be missed by contemporary practice where somatic mutations are being filtered against the germline to remove non-functional SNPs.

\section{Conclusion}

In conclusion, potential germline mutations affecting the CHEK2 gene should be taken into consideration when exploring the genetic mechanisms of drug resistance among breast and, most likely, also prostatic cancer patients.

\begin{abstract}
Acknowledgements
Not applicable.

Funding

The present study was supported by grants from the Norwegian Cancer Society, the Norwegian Research Council, the Bergen Research Foundation and the Norwegian Health Region West. The funding bodies did not have any saying with regards to study design, collection, analysis or interpretation of data, or in writing of the manuscript.
\end{abstract}

Availability of data and materials

Please contact author for data requests.

\section{Authors' contributions}

SK conceived the study, performed data analyses and wrote the manuscript. BL performed laboratory analyses. LBG designed and supervised laboratory analyses. PR contributed samples and statistical advice. KH contributed samples. LV contributed samples and statistical advice. PEL conceived the study and wrote the manuscript. All authors read and approved the final manuscript. 


\section{Competing interests}

The authors declare that they have no competing interests.

\section{Consent for publication}

Not applicable.

\section{Ethics approval and consent to participate}

The study was approved by the Regional Ethics Committee of Mid-Norway (REK, Helse Midt-Norge). All participants provided written informed consent.

\section{Author details}

'Section of Oncology, Department of Clinical Science, University of Bergen, 5020 Bergen, Norway. ${ }^{2}$ Department of Oncology, Haukeland University Hospital, Bergen, Norway. ${ }^{3}$ Department of Public Health, Faculty of Medicine, Norwegian University of Science and Technology, Trondheim, Norway.

Received: 13 March 2016 Accepted: 21 September 2016

Published online: 27 September 2016

\section{References}

1. Cybulski C, Huzarski T, Gorski B, Masojc B, Mierzejewski M, Debniak T, et al. A novel founder CHEK2 mutation is associated with increased prostate cancer risk. Cancer Res. 2004;64:2677-9.

2. Cybulski C, Wokolorczyk D, Jakubowska A, Huzarski T, Byrski T, Gronwald $J$, et al. Risk of breast cancer in women with a CHEK2 mutation with and without a family history of breast cancer. J Clin Oncol Off J Am Soc Clin Oncol. 2011;29:3747-52.

3. Meijers-Heijboer $H$, van den Ouweland A, Klijn J, Wasielewski M, de Snoo A, Oldenburg R, et al. Low-penetrance susceptibility to breast cancer due to CHEK2 $\left.{ }^{*}\right) 1100$ delC in noncarriers of BRCA1 or BRCA2 mutations. Nat Genet. 2002;31:55-9.

4. Krivokuca A, Dobricic J, Brankovic-Magic M. CHEK2 1100delC and Del5395bp mutations in BRCA-negative individuals from Serbian hereditary breast and ovarian cancer families. J BUON. 2013;18:594-600.

5. Walsh T, Casadei S, Coats KH, Swisher E, Stray SM, Higgins J, et al. Spectrum of mutations in BRCA1, BRCA2, CHEK2, and TP53 in families at high risk of breast cancer. JAMA. 2006;295:1379-88.

6. Havranek O, Kleiblova P, Hojny J, Lhota F, Soucek P, Trneny M, et al. Association of germline CHEK2 gene variants with risk and prognosis of Non-Hodgkin lymphoma. PLoS One. 2015;10:e0140819.

7. Siolek M, Cybulski C, Gasior-Perczak D, Kowalik A, Kozak-Klonowska B, Kowalska A, et al. CHEK2 mutations and the risk of papillary thyroid cancer. Int J Cancer. 2015;137:548-52.

8. Chrisanthar R, Knappskog S, Lokkevik E, Anker G, Ostenstad B, Lundgren S, et al. CHEK2 mutations affecting kinase activity together with mutations in TP53 indicate a functional pathway associated with resistance to epirubicin in primary breast cancer. PLOS ONE. 2008;3:e3062.

9. Knappskog S, Berge EO, Chrisanthar R, Geisler S, Staalesen V, Leirvaag B, et al. Concomitant inactivation of the p53- and pRB- functional pathways predicts resistance to DNA damaging drugs in breast cancer in vivo. Mol Oncol. 2015;9:1553-64

10. Knappskog S, Chrisanthar R, Lokkevik E, Anker G, Ostenstad B, Lundgren $\mathrm{S}$, et al. Low expression levels of ATM may substitute for CHEK2 /TP53 mutations predicting resistance towards anthracycline and mitomycin chemotherapy in breast cancer. Breast Cancer Res. 2012;14:R47.

11. Naess O, Sogaard AJ, Arnesen E, Beckstrom AC, Bjertness E, Engeland A, et al. Cohort profile: cohort of Norway (CONOR). Int J Epidemiol. 2008;37:481-5.

12. Gansmo LB, Knappskog S, Romundstad P, Hveem K, Vatten L, Lonning PE. Influence of MDM2 SNP309 and SNP285 status on the risk of cancer in the breast, prostate, lung and colon. Int J Cancer. 2015;137:96-103.

13. Genomes Project C, Abecasis GR, Auton A, Brooks LD, DePristo MA, Durbin RM, et al. An integrated map of genetic variation from 1,092 human genomes. Nature. 2012:491:56-65

14. Huijts PE, Hollestelle A, Balliu B, Houwing-Duistermaat JJ, Meijers CM, Blom JC, et al. CHEK2*1100delC homozygosity in the Netherlands-prevalence and risk of breast and lung cancer. Eur J Hum Genet. 2014;22:46-51.
15. Wang N, Ding H, Liu C, Li X, Wei L, Yu J, et al. A novel recurrent CHEK2 Y390C mutation identified in high-risk Chinese breast cancer patients impairs its activity and is associated with increased breast cancer risk. Oncogene. 2015:34:5198-205.

16. Staalesen V, Falck J, Geisler S, Bartkova J, Borresen-Dale AL, Lukas J, et al. Alternative splicing and mutation status of CHEK2 in stage III breast cancer. Oncogene. 2004;23:8535-44.

\section{Submit your next manuscript to BioMed Central and we will help you at every step:}

- We accept pre-submission inquiries

- Our selector tool helps you to find the most relevant journal

- We provide round the clock customer support

- Convenient online submission

- Thorough peer review

- Inclusion in PubMed and all major indexing services

- Maximum visibility for your research

Submit your manuscript at www.biomedcentral.com/submit
Biomed Central 